\title{
Collecting of finely dispersed particles by means of a separator with the arc-shaped elements
}

\author{
Andrey V. Dmitriev ${ }^{1, *}$, Vadim E. Zinurov ${ }^{1}$, and Oksana S. Dmitrieva ${ }^{2}$ \\ ${ }^{1}$ Kazan State Power Engineering University, 420066, Kazan, Krasnoselskaya str., 51, Russia \\ ${ }^{2}$ Kazan National Research Technological University, 420015, Kazan, Karl Marx str., 68, Russia
}

\begin{abstract}
This paper includes the description of a separator, developed by the authors for the gas flow purification from the finely dispersed particles. The authors also studied the influence of the separator dimensions and the dusty gas flow rate on the degree of its purification from the finely dispersed particles, as well as on the change in the hydraulic resistance of this apparatus. This paper also shows that the main forces that make the greatest contribution to the purification of the gas flow from the finely dispersed particles are centrifugal and inertial. Moreover, the dependencies of the purification efficiency on the Stokes numbers are shown in this paper. The authors studied the change in the hydraulic resistance coefficient of this apparatus from the Reynolds numbers as well.
\end{abstract}

\section{Introduction}

Nowadays, the purification of the gas flows from the finely dispersed particles with a diameter of less than $20 \mu \mathrm{m}$ is an urgent task for the foundry, petrochemical, chemical, metallurgical and other industries. For example, the solid foundry wastes contain up to $90 \%$ of the spent molding and core sand mixtures, including the defect formworks and rods. They also contain the foundry slag and abrasive dust, refractory materials and ceramics. In order to collect these particles, the foundry industrial plants can use the following apparatuses in a unique technological scheme for the air purification: inertial dust collectors, fabric filters, electrostatic dust collectors, etc. [1-2].

In order to purify the air (technological purification cycle), as a rule, the following equipment is installed first: vertical dust-collecting chambers, fly-ash collectors or cyclones, allowing to separate the coarse dust from the gas flows and significantly reduce the dustiness of the gaseous medium. After that, the fabric bag filters or electrostatic dust collectors are to be installed that effectively purify the gas flows from the particles with a diameter of less than $20 \mu \mathrm{m}$ [3-8].

However, as they operate, the gas purification efficiency decreases significantly. The bag filters require periodic replacement of elements that is a cost-intensive measure, while electrostatic dust collectors need to be cleaned that is a time-taking process. To install the

\footnotetext{
*Corresponding author: ieremiada@gmail.com
} 
bag filter is about 1.5-2 times cheaper than the electric filters, but operation is more expensive. Generally, the foundry industrial plants spend significant economical means for purchasing and operating the bag filters and electrostatic dust collectors [9-14]. Therefore, the development of new technological apparatuses, allowing to increase the efficiency and to reduce the costs for the gas purification from various finely dispersed particles, is one of the solutions to this problem.

\section{Solution to the problem}

The authors of this paper developed a separator, allowing to clean the gas flows from the finely dispersed particles with a diameter of less than $10 \mu \mathrm{m}$ with an efficiency of at least $50 \%$ and when the diameter is more than $12 \mu \mathrm{m}$ - the efficiency is at least $99 \%$ at an input gas flow rate of over $5 \mathrm{~m} / \mathrm{s}$. $995 \mathrm{~Pa}$ is the pressure loss value in the apparatus when the efficiency is equal to $1.6 \mathrm{~m}^{3} / \mathrm{s}$. It is advisable to install this apparatus in front of the bag or electric filters, that will extend the service life of them by 2 or more times.

The developed apparatus consists of several rows of the arc-shaped elements, arranged in a rectangular case. The design of the separator includes inlet and outlet nozzles, as well as a hopper. The arc-shaped elements are located in the separator at an angle of $30^{\circ}$ to the basement in order to create a constant gas flow rate and to ensure minimum hydraulic resistance (Fig. 1).

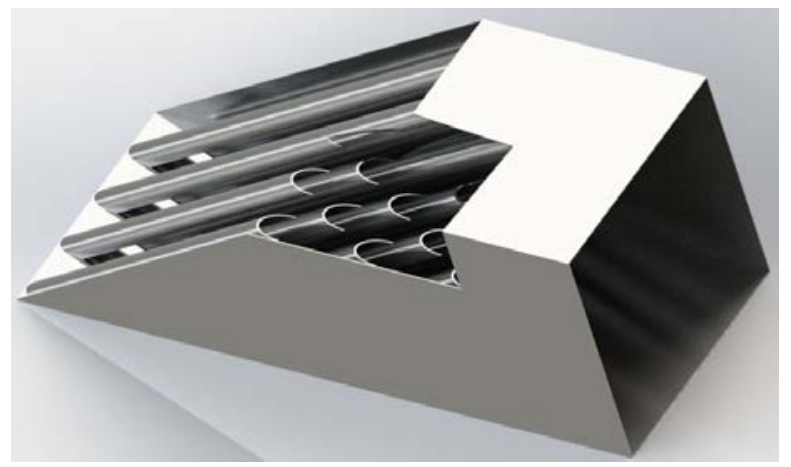

Fig. 1. 3D model of a rectangular separator (section view).

The operating principle of this separator is the following: the dusty gas flow enters the apparatus through the inlet nozzle, then it goes round the several rows of the arc-shaped elements, while the centrifugal force affects it and contributes to deposion of the dust particles from a structured flow. After that, the purified gas leaves the apparatus through the outlet nozzle. Settling out of small particles on the surfaces of the arc-shaped elements can also be a result of electrostatic forces, Brownian motion, inertial forces and the combination of all these factors [15-18]. The finely dispersed particles settle on the surfaces of the elements, which are periodically cleaned by means of special equipment (Fig. 2).

Due to the fact that the purification of the gas flow in the separator is carried out mainly by centrifugal forces, the arc-shaped elements are installed in the apparatus so that its maximum possible value is achieved when the gas moves between the elements.

\section{Purpose of the study}

The purpose of this study is to analyze the influence of the rectangular separator dimensions and the rate of the dusty gas on the degree of its purification from the dust particles, as well as on the change in the hydraulic resistance of the apparatus. 


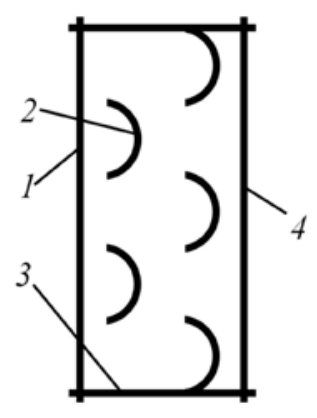

Fig. 2. Simplified 2D model of a rectangular separator (top view perpendicular to the arc-shaped elements): 1 -inlet nozzle; 2 - arc-shaped elements inside of the separator; 3 - case of the apparatus; 4 - outlet nozzle.

In order to calculate the rectangular separator, the finite-element method in ANSYS Fluent software program was applied, as well as the turbulence model - SST was used.

In the course of study, the following parameters were set: atmospheric pressure at the outlet of the apparatus $-101325 \mathrm{~Pa}$, initial rate of the particles in the gas flow $-0 \mathrm{~m} / \mathrm{s}$, number of particles in the gas $n-1000$, density of the gas flow $\rho-1.22 \mathrm{~kg} / \mathrm{m}^{3}$, coefficients of kinematic $v$ and dynamic $\mu$ gas viscosity $-1 \cdot 10^{-6} \mathrm{~m}^{2} / \mathrm{s}$ and $18.1 \cdot 10^{-6} \mathrm{~Pa} \cdot \mathrm{s}$. In order to obtain the results, the following parameters were changed: the rate at the inlet of the apparatus $W$ within the range of $1-15 \mathrm{~m} / \mathrm{s}$, the diameter of particles a in the gas flow was taken from 1 to $15 \mu \mathrm{m}$. In the course of study, the dimensions of the rectangular separator were changed, namely the section of the inlet nozzle varied from $0.1 \times 0.1$ to $0.4 \times 0.4 \mathrm{~m}^{2}$. In proportion to the change in dimensions of the rectangular separator, all structural elements were changed: the length of the arc-shaped elements was taken within the range of 12.5-50 $\mathrm{mm}$. During each study, the number of rows of the arc-shaped elements inside of the separator remained unchanged -5 . The study was carried out at different capacity of the rectangular separator within the range of $0.01-2.4 \mathrm{~m}^{3} / \mathrm{s}$. In order to simplify the calculations, the wall thickness in this study was not taken into account; it was set to an infinitely small value. When carrying out the numerical simulation, the adhesion condition was set.

The numerical calculation was carried out under the following assumptions: the gas flow process was stationary, the dust concentration excluded the interaction between the particles, and the influence of particles on the motion of the carrier medium was not taken into account.

In the course of studies, the efficiency E of the gas flow purification from the finely dispersed dust particles by means of the rectangular separator was determined:

$$
E=1-\frac{n_{k}}{n}
$$

where $n_{k}$ - the number of particles, left in the gas flow after the purification process in rectangular separator, $n$ - number of particles, available in the gas.

Pressure values were measured at the inlet and outlet nozzles of the apparatus. As a result, the hydraulic resistance coefficient $\xi$ of the rectangular separator was calculated:

$$
\xi=\frac{2 h}{H} \frac{\Delta p}{\rho W_{h}^{2}},
$$


where $\Delta p$ - pressure losses in the separator, $\mathrm{Pa} ; H$ - distance between the first and the last row of the arc-shaped elements, $\mathrm{m} ; h$ - radius of the circles around elements, $\mathrm{m} ; \rho$ - gas density, $\mathrm{kg} / \mathrm{m}^{3} ; W_{h}$ - rate within the narrowing parts of the separator, $\mathrm{m} / \mathrm{s}$.

The Reynolds number $(\mathrm{Re})$ was determined by the following formula:

$$
\operatorname{Re}=\frac{a W_{h}}{v},
$$

where $a$-diameter of the particles, $\mathrm{m} ; \mathrm{v}$ - coefficient of kinematic viscosity, $\mathrm{m}^{2} / \mathrm{s}$.

The ratio between the kinetic energy of suspended particles and the energy of their interaction with the gas was determined by means of the Stokes number:

$$
\mathrm{Stk}=\frac{\rho_{a} a^{2} W_{h}}{\mu h},
$$

where $\rho_{a}$ - particle density, $\mathrm{kg} / \mathrm{m}^{3} ; \mu$ - coefficient of dynamic viscosity of gas, Pa.s.

Due to the fact that the arc-shaped elements were placed against each other and the inlet nozzle as per the equal flow principle, then the rate within the narrowing parts of rectangular separator $W_{h}$, as in the Eqs. (2)-(4), was taken as equal to the rate of the gas flow $W$ at the inlet of the apparatus.

\section{Results of studies and clarification of them}

The results of the studies are shown graphically in Fig. 3-7. Numerical studies show that the gas flow purification from the finely dispersed particles with a diameter of more than 9 $\mu \mathrm{m}$ is carried out with an efficiency higher than $90 \%$, and for the particles with a diameter of less than $9 \mu \mathrm{m}$, the gas purification value is at least $55 \%$. With an increase in the input the gas flow rate, the efficiency of collecting the dust particles with a diameter of less than $7 \mu \mathrm{m}$ from the gas flow increases. For the particles with a diameter of more than $7 \mu \mathrm{m}$, the gas purification efficiency is not significant when the rate changes. However, this is true for a rectangular separator with section on the inlet nozzle of $0.1 \times 0.1 \mathrm{~m}$. As the dimensions of the apparatus increase, the gas purification degree also increases for the particles of any size with an increase in the input rate $W$.

As noted earlier, the greatest contribution to the purification of the gas flow from the finely dispersed particles is made by the centrifugal force, which appears when the gas goes round the arc-shaped elements. Therefore, it can be assumed that the degree of purification by means of two rows of the arc-shaped elements is significantly lower than by the other rows, since this case the main force, affecting the gas purification, is inertial one. As a result, the concept of a purifying stage was introduced by the authors. The area of a row of the arc-shaped elements is a purifying stage itself, where the gas flow is purified under the influence of centrifugal forces. Therefore, when calculating the efficiency of purifying stage, it is necessary to subtract the first two rows of the arc-shaped elements. In order to confirm this assumption, the authors calculated the efficiency of the first two rows $E_{0}$ and the purifying stage $E_{1}$.

The efficiency of the first two rows $E_{0}$ was determined by the following formula:

$$
E_{0}=\frac{n_{0}}{n}
$$

where $n_{0}$ - the number of particles, remained in the first row of the arc-shaped elements of the rectangular separator. 
Operating efficiency of the purifying stage $E_{1}$ was determined by the following formula:

$$
E_{1}=1-\left(\frac{1-E}{1-E_{0}}\right)^{\frac{1}{m-2}}
$$

where $m$ - number of rows of the arc-shaped elements within a rectangular separator.

In the course of comparing the efficiency values of the gas flow purification from the finely dispersed particles by means of purifying stage and the first two rows of the arcshaped elements, it was found that the stage purifies the gas on average by 4.54 times more effectively than the first two rows of the elements at an input gas flow rate of $15 \mathrm{~m} / \mathrm{s}$. On average, the efficiency of the first two rows of the arc-shaped elements is $13.6 \%$, the average efficiency of each purifying stage is $61.8 \%$. By increasing the size of the inlet section of nozzle from $0.1 \times 0.1$ to $0.4 \times 0.4 \mathrm{~m}^{2}$, the efficiency of collecting the particles from the gas flow by means of the purifying stage decreases on average by $19.1 \%$. By increasing the size of the separator, the efficiency of collecting the particles from the gas flow by means of the first row of the arc-shaped elements decreases on average by $5 \%$. The average efficiency values of the gas purification from the finely dispersed particles at the following size of the inlet section of nozzle $-0.1 \times 0.1,0.2 \times 0.2$, and $0.4 \times 0.4 \mathrm{~m}^{2}$ are equal to $71.1 \%$, $62.1 \%$ and $52.3 \%$, respectively (Fig. 3).

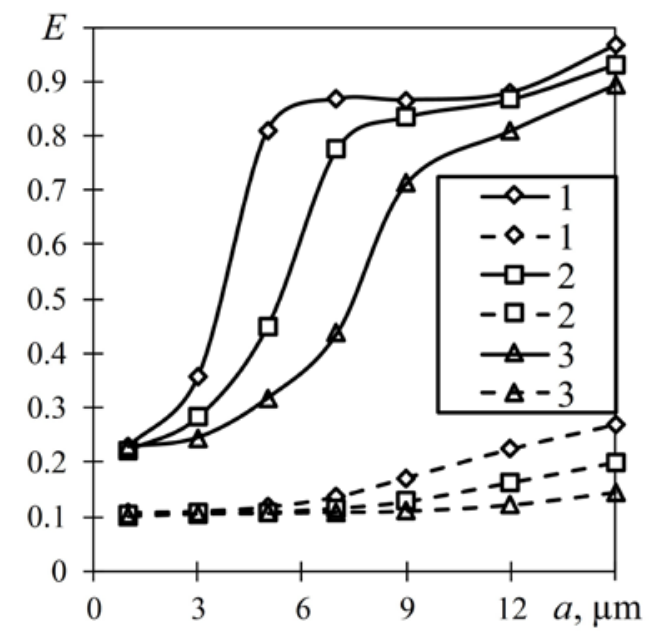

Fig. 3. Dependency of change in efficiency of the gas flow purification on the diameter of the finely dispersed particles at the input gas flow rate of $15 \mathrm{~m} / \mathrm{s}$ and at different sizes of the inlet nozzle, $\mathrm{m}^{2}$ : $1-0.1 \times 0.1 ; 2-0.2 \times 0.2 ; 3-0.4 \times 0.4$. The solid line shows the operation efficiency of the purifying stage, dashed lines show the efficiency of the first row of the arc-shaped elements.

The maximum efficiency of the gas flow purification from the finely dispersed particles by rectangular separator is achieved when the Stokes number values are over 1.6. On average, the efficiency of the gas flow purification is equal to $98.2 \%$ when the Stokes numbers are over 1.6. If the Stokes numbers are less than 1.6, the efficiency significantly reduces. This is due to the fact that these Stokes numbers were calculated at unfavourable parameters: relatively low input gas flow rates, large dimensions of the apparatus, etc. The average gas purification efficiency when the Stokes numbers are less than 1.6, was equal to $69.4 \%$ (Fig. 4). 


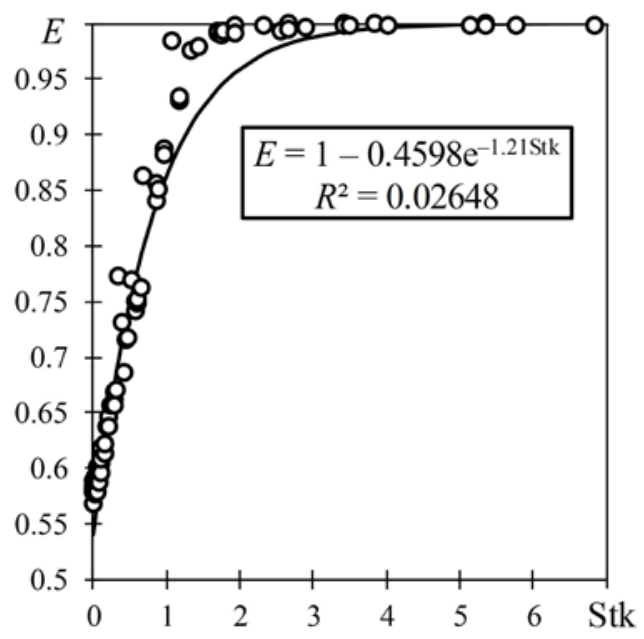

Fig. 4. Dependency of change in efficiency of the gas flow purification from the finely dispersed particles by the rectangular separator on the Stokes numbers.

Moreover, the dependences of the efficiency of the gas purification from dust by means of the purifying stage on the Stokes numbers, as well as dependences of the efficiency of the gas purification by the first row of the arc-shaped elements on the Stokes numbers, were obtained (Fig. 5 and 6).

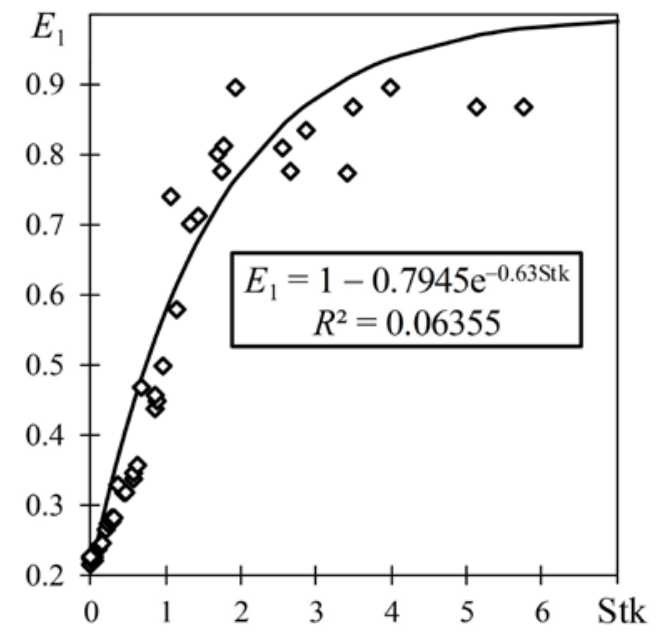

Fig. 5. Dependency of change in efficiency of the gas flow purification from the finely dispersed particles by the purifying stage of the rectangular separator on the Stokes numbers.

The maximum efficiency of the gas flow purification from the finely dispersed particles by means of the purifying stage of the rectangular separator is achieved when the Stokes numbers are over 0.9. At these values of the Stokes numbers, the efficiency of the gas flow purification is equal on average to $77.9 \%$. When the Stokes number values are less than 0.9 , the efficiency of collecting the particle from the gas flow by means of the purifying stage reduces to $29.1 \%$ (Fig. 5). 


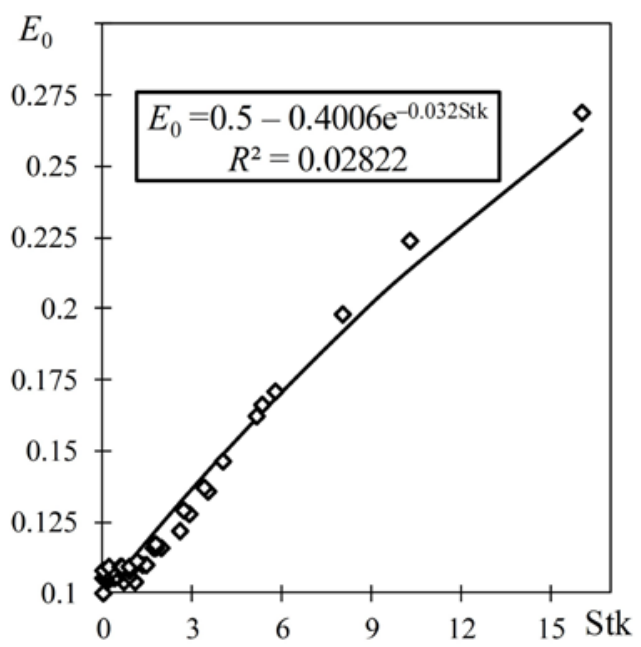

Fig. 6. Dependency of change in efficiency of the gas flow purification from the finely dispersed particles by the first row of the arc-shaped elements of the rectangular separator on the Stokes numbers.

Practically all values of the gas flow purification efficiency by the first row of the arcshaped elements are within the range of the Stokes numbers from 0 to 6 . The average efficiency of the gas flow purification by the first row of the arc-shaped elements for this range is equal to $11.4 \%$. For the Stokes numbers over 6 , the average efficiency is equal to $13.2 \%$. It is worth noting that the obtained equation of dependency of the gas purification efficiency by the first row of the arc-shaped elements on the Stokes numbers, shown in Fig. 6 , differs from the previous equations by a coefficient equal to 0.5 .

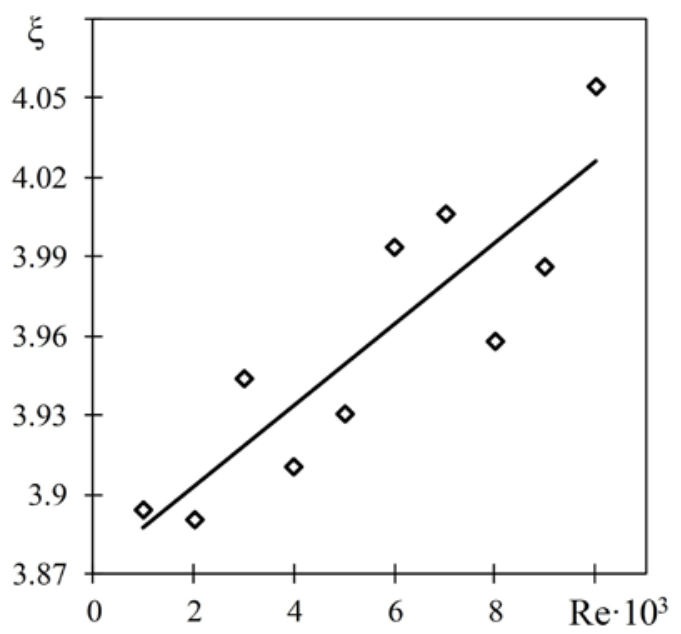

Fig. 7. Dependency of change in the hydraulic resistance coefficient on the Reynolds number.

This is due to the following factors: the first row covers $50 \%$ of the area of movement of the dusty gas flow and, the condition of adhesion was also initially set (Fig. 6). The hydraulic resistance coefficient of the rectangular separator on average is equal to 3.96 . Mean-root-square error for this study is equal to $3.31 \%$ (Fig. 7). 


\section{Conclusion}

In the course of studies, it was found that the rectangular separator effectively purifies the gas flow from the finely dispersed particles with a sufficiently low hydraulic resistance coefficient. The studies confirmed that the developed apparatus has dominated action of centrifugal forces, which are more effective than other forces: inertial, electrostatic, etc. when purifying the dusty gas. Equations of dependencies of efficient gas flow purification on the Stokes numbers, as well as the hydraulic resistance coefficient - on the Reynolds numbers, were obtained. Co-solution of these equations allows to calculate the required input gas flow rate, at a certain length of the arc-shaped elements, in order to achieve the maximum efficiency of the gas flow purification from the dust particles, while the hydraulic resistance is minimum, or to carry out this calculation in the reverse order. The studies showed that the most effective input rate of the gas flow is within the range of 10$12 \mathrm{~m} / \mathrm{s}$. The advantages of the rectangular separator are the following: low metal consumption, high efficiency of the gas flow purification from the finely dispersed particles, simplicity of design, lack of moving elements.

The study was performed with funding from the RF President`s grant project No. MK-4522.2018.8.

\section{References}

1. P.A. Patterson, R.J. Munz, Can. J. Chem. Eng., 74, 213-221 (1996)

2. S.M. Clifford, J. Zhang, T. Sigsgaard, M. Jantunen, P.J. Lioy, R. Samson, M.H. Karol, Environ. Health Perspect., 115, 958-964 (2007)

3. P. Baltrenas, M. Pranskevicius, A. Venslovas, Energy Procedia, 72, 188-195 (2015)

4. S. Bernardo, M. Mori, A.P. Peres, R.P. Dionisio, Powder Technol., 162, 190-200 (2006)

5. S. Altmeyer, V. Mathieu, S. Jullemier, P. Contal, N. Midoux, S. Rode, J.P. Leclers, Chem. Eng. Prog, 43, 511-522 (2004)

6. B. Wang, D.L. Xu, K.W. Chu, A.B. Yu, Appl. Math. Modelling, 30, 1326-1342 (2006)

7. Y. Su, A. Zheng, B. Zhao, Powder Technol., 210, 293-303 (2011)

8. A.V. Dmitriev, V.E. Zinurov, O.S. Dmitrieva, IOP Conf. Ser.: Mater. Sci. Eng., 451, 012211 (2018)

9. R. Singh, A. Shukla, Renewable Sustainable Energy Rev., 40, 118-132 (2014)

10. G. Guan, Chin. J. Chem. Eng., 25, 689-697 (2017)

11. Y.-S. Chen, S.-S. Hsiau, J.-R. Syu, Y.-L. Chang, Fuel, 248, 136-142 (2019)

12. A.V. Dmitriev, I.N. Madyshev, O.S. Dmitrieva, Lecture Notes in Mechanical Engineering (Springer, Cham, 2019)

13. O.V. Soloveva, S.A. Solovev, R.R. Khusainov, A.S. Shubina, J. Phys.: Conf. Ser. 1158, 042023 (2019)

14. N.A. Merentsov, V.A. Balashov, S.A. Bokhan, E.E. Nefed'eva, D.A. Tezikov, V.V. Groshev, IOP Conf. Ser.: Earth Environ. Sci., 224, 012041 (2019)

15. J. Chang, P. Wang, L. Cui, Z. Li, C. Ma, X. Wang, J. Electrost., 95, 53-60 (2018)

16. S. Koshkarev, A. Evtushenko, S. Pushenko, Procedia Eng., 165, 1057-1069 (2016)

17. B.K. Lee, K.R. Jung, S.H. Park, J. Aerosol Sci., 12, 1079-1088 (2008)

18. O.S. Makusheva, A.V. Dmitriev, N.A. Nikolaev, Chem. Pet. Eng., 46, 330-333 (2010) 